\title{
Spatially Coupled LDPC Codes and the Multiple Access Channel
}

\author{
(Invited Paper)
}

\author{
Sebastian Cammerer, Xiaojie Wang, Yingyan Ma, and Stephan ten Brink \\ Institute of Telecommunications, Pfaffenwaldring 47, University of Stuttgart, 70569 Stuttgart, Germany \\ \{cammerer,wang,tenbrink\}@inue.uni-stuttgart.de
}

\begin{abstract}
We consider spatially coupled low-density paritycheck (SC-LDPC) codes within a non-orthogonal interleave division multiple access (IDMA) scheme to avoid cumbersome degree profile matching of the LDPC code components to the iterative multi-user detector (MUD). Besides excellent decoding thresholds, the approach benefits from the possibility of using rather simple and regular underlying block LDPC codes owing to the universal behavior of the resulting coupled code with respect to the channel front-end, i.e., the iterative MUD. Furthermore, an additional outer repetition code makes the scheme flexible to cope with a varying number of users and user rates, as the SC-LDPC itself can be kept constant for a wide range of different user loads. The decoding thresholds are obtained via density evolution (DE) and verified by bit error rate (BER) simulations. To keep decoding complexity and latency small, we introduce a joint iterative windowed detector/decoder imposing carefully adjusted sub-block interleavers. Finally, we show that the proposed coding scheme also works for Rayleigh channels using the same code with tolerable performance loss compared to the additive white Gaussian noise (AWGN) channel.
\end{abstract}

\section{INTRODUCTION}

While in the single-user case the Shannon capacity has been almost achieved for practical coding schemes [1], [2], the situation changes when considering the multi-user nonorthogonal multiple access (NOMA) scenario, i.e., when multiple transmitters and a single receiver share the same medium [3]. Although several NOMA approaches exist (see [4], [5] and references therein), it is still an open and interesting research direction to find low-complexity coding (and detection) schemes that operate close to the multi-user capacity. Further, multi-user systems open up yet unsettled research opportunities such as the flexibility towards a dynamically varying number of users and, likely, having different power levels.

One attractive NOMA scheme, featuring low-complexity, parallelizable computation and asynchronous transmission, is interleave division multiple access (IDMA) [6], [7]. In this work, we focus on the IDMA scheme, where a low-complexity parallel interference cancellation (PIC) receiver is used and an effective separation between users is done by an individual interleaver. Relying on feedback from single-user channel decoders, the performance of IDMA systems strongly depends on the performance of the underlying channel codes.
Thus, in classical low-density parity-check (LDPC)-based IDMA systems, the LDPC code needs to have matched degree profiles [8], [9] to the channel front-end, i.e., the multi-user detector (MUD). As this optimization depends on several parameters such as the channel type, the number of users and the individual signal-to-noise ratios (SNRs), the drawback is that in practice either multiple LDPC codes need to be (pre-)designed, or a degraded system performance has to be accepted. In this work, we make use of the fact that spatially coupled low-density parity-check (SC-LDPC) codes do not have this drawback as they are known for a universal behavior regarding the channel front-end [10], [11].

SC-LDPC codes are widely known for their capacity achieving decoding behavior via threshold saturation [12]. More precisely, it has been shown that coupled codes approach the maximum a posteriori (MAP) decoding performance of the underlying block LDPC code under low-complexity belief propagation (BP) decoding for properly chosen code parameters. However, a second powerful property of SC-LDPC codes is not so often referred to, but can be seen in their universality with respect to the channel front-end, i.e., for carefully chosen code parameters SC-LDPC codes do not need any re-design when the channel characteristics change. It also offers potentially low error-floors as typically a regular code design suffices which simplifies the code construction. This universality renders SC-LDPC codes into a promising candidate for NOMA schemes where a wide range of different scenarios must be supported such as different number of users, channels and user power.

Unfortunately, as SC-LDPC codes are constructed out of multiple coupled sub-blocks, they typically introduce long block lengths. Thus, for practical decoder implementations, i.e., feasible decoding complexity, a windowed decoder is crucial [13]. We show that the MUD can be integrated into the iterative detection/decoding scheme with negligible performance loss. Yet, this requires some attention with respect to the decoding window and the interleaver design to separate individual users, as the iterative detection/decoding loop needs to be performed sub-block-wise to maintain the benefits of threshold saturation. 

SC-LDPC Encoder
REP Encoder
Interleaver
Mapper
and Channel
Phase-Scrambler

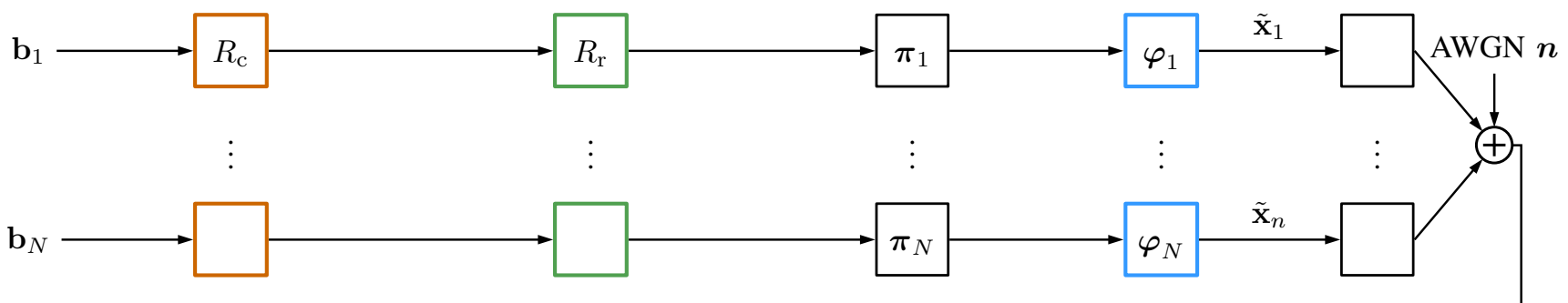

SC-LDPC Decoder REP Decoder Deinterleaver $\quad \begin{gathered}\text { Demapper } \\ \text { and }\end{gathered}$

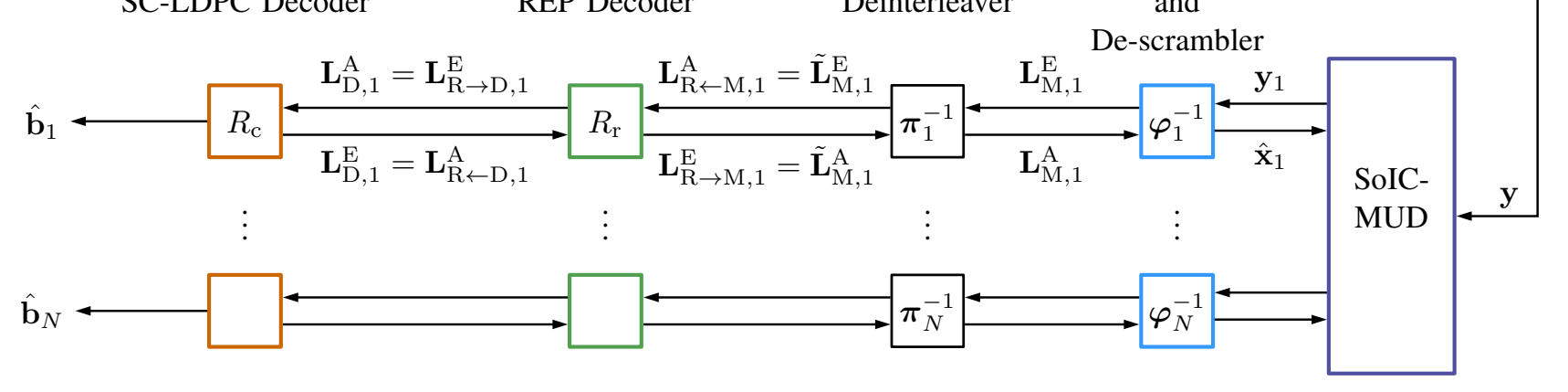

Fig. 1. IDMA system model; all users have the same coding and modulation scheme; note that boldface letters denote vectors. The receiver can be implemented in a windowed version (see Sec. IV for details), however, for simplicity indices related to windowed decoding are omitted.

\section{IDMA SYSTEM MODEL}

Fig. 1 shows the IDMA system model with $N$ uncooperative users. Each user encodes and decodes its data separately using a channel encoder (SC-LDPC code here) of code rate $R_{c}$ and a common serially concatenated repetition code of rate $R_{r}=\frac{1}{d_{\mathrm{r}}}$ (see [9] for details). Note that the SC-LDPC code and, thus, also the code parameters, e.g., degree profile and coupling width $W$, are the same among all the $N$ users. Thus, the total code-rate is $R_{\mathrm{tot}}=R_{c} R_{r}$. The interleaver is, on the contrary, user-specific to allow efficient user separation at the receiver. After interleaving, the coded bits are mapped to symbols, e.g., using binary phase shift keying (BPSK), and transmitted over the Gaussian multiple access channel (GMAC).

The $m$ th received signal (i.e., the $m$ th element of $\mathbf{y}$ in Fig. 1) of all users can be written as

$$
y_{m}=\sum_{i=1}^{N} \sqrt{P_{i}} h_{i, m} \cdot \underbrace{x_{i, m} \cdot e^{j \varphi_{i, m}}}_{:=\widetilde{x}_{i, m}}+n_{m}
$$

where $m$ is the discrete-time index, $\sqrt{P_{i}}$ denotes the signal power of the $i$ th user, $h_{i, m}$ is the uncorrelated (both over time and among different users) small-scale Rayleigh fading channel coefficient, $n_{m}$ is circularly symmetric (complexvalued) AWGN with zero mean and variance $\sigma_{n}^{2}$, and $\varphi_{i, m}$ is a pseudo random phase scrambling to avoid ambiguity of the super-constellation (Cartesian product of all user constellations). This random phase shift could also be the consequence of, e.g., the channel and/or explicit "scrambling" and we include this into each user's mapper (only in AWGN channels; for Rayleigh channels this step can be omitted). Throughout this paper, the phases $\varphi_{i, m}$ are independently and uniformly distributed in $[0, \pi)$. The output of the mapper of the $i$ th user with BPSK modulation at the $m$ th time instant is $\tilde{x}_{i, m} \in\left\{ \pm e^{j \varphi_{i, m}}\right\}$. The phase scrambling can improve the superimposed multiuser codeword distance [14], particularly in AWGN channels. The so-called multi-user SNR is defined as

$$
\gamma=\frac{\sum_{i=1}^{N} P_{i}}{\sigma_{n}^{2}} .
$$

The received signal is first processed by a multi-user detector (MUD). An optimum MUD is to compute the maximum a posteriori (MAP) probability of each bit. This requires a complexity of $O\left(M^{N}\right)$ where $M$ denotes the number of constellation symbols per user. The exponentially increasing complexity with the number of users $N$ prohibits its practical implementation for a large number of users. Therefore, a sub-optimal soft interference cancellation (SoIC) based low complexity MUD was proposed in [6]. The sub-optimal MUD first cancels out the other users' signals; for instance, the $i$ th user's signal is estimated by the conditional minimum meansquare error (MMSE) estimator for BPSK

$$
\hat{x}_{i}=\tanh \left(\frac{L_{\mathrm{M}, i}^{\mathrm{A}}}{2}\right) \cdot e^{j \varphi_{i}}
$$

based on, e.g., the a posteriori knowledge of the channel decoder $L_{\mathrm{M}, i}^{\mathrm{A}}$ (the SC-LDPC decoder output is $L_{\mathrm{R} \leftarrow \mathrm{D}, i}^{\mathrm{A}}$; it is 
then re-encoded by a repetition code (REP) and re-interleaved with the outputs denoted by $\tilde{L}_{\mathrm{M}, i}^{\mathrm{A}}$ and $L_{\mathrm{M}, i}^{\mathrm{A}}$, respectively). For an arbitrary user $j$ (the symbol index $m$ is dropped for brevity), the output of the MUD after the SoIC can be written as

$$
y_{j}=\sqrt{P_{j}} h_{j} \widetilde{x}_{j}+\sum_{i=1, i \neq j}^{N} \sqrt{P_{i}} h_{i}\left(\tilde{x}_{i}-\hat{x}_{i}\right)+n .
$$

Then, each user starts its single user detection and decoding in parallel. The (soft) demapper computes the log-likelihoodratio (LLR) of each bit while treating the residual interference as noise. For BPSK, an approximation of the true a posteriori LLR can be computed according to

$$
L_{\mathrm{M}, j}^{\mathrm{E}}=4 \sqrt{P_{j}} \frac{\operatorname{Re}\left\{y_{j} \cdot h_{j}^{*} \cdot e^{-j \varphi_{j}}\right\}}{\sigma_{\mathrm{I}, j}^{2}+\sigma_{n}^{2}}
$$

where the noise variance $\sigma_{n}^{2}$, the random phase shifts $\varphi_{j}$ and the channel coefficients $h_{j}$ are assumed to be known at the receiver. The interference power can be estimated by

$$
\begin{aligned}
& \sigma_{\mathrm{I}, j}^{2}=\mathrm{E}\left[\left|e^{-j \varphi_{j}} \sum_{i \neq j} \sqrt{P_{i}} h_{i}\left(\tilde{x}_{i}-\hat{x}_{i}\right)\right|^{2}\right] \\
= & \sum_{i \neq j} P_{i}\left|h_{i}\right|^{2}\left(1-\mathrm{E}\left[\tanh \left(\frac{L_{\mathrm{M}, i}^{\mathrm{E}}}{2}\right)\right]^{2}\right)
\end{aligned}
$$

where the interference term is assumed to be Gaussian distributed, provided that the number of users $N$ is large enough and the transmitted symbols are independent among users (central limit theorem).

Then, the LLRs are deinterleaved (denoted by $\tilde{L}_{\mathrm{M}, j}^{\mathrm{E}}=$ $L_{\mathrm{R} \leftarrow M, j}^{\mathrm{A}}$ which means the extrinsic message from the MUD corresponds to the a priori knowledge of the REP obtained by the MUD) and sent to a repetition decoder. The extrinsic message from the repetition code to the LDPC decoder is given by

$$
L_{\mathrm{D}, j, m}^{\mathrm{A}}=L_{\mathrm{R} \rightarrow \mathrm{D}, j, m}^{\mathrm{E}}=\sum_{k=m d_{\mathrm{r}}}^{(m+1) d_{\mathrm{r}}-1} \tilde{L}_{\mathrm{M}, j, k}^{\mathrm{E}} .
$$

Subsequently, channel decoding can be performed by the corresponding channel decoder.

\section{Spatially Coupled LDPC}

We follow the definitions in [15], i.e., consider SC-LDPC code ensembles defined by their protograph matrix B. Protographs can be seen as a blueprint of larger graphs, where $S$ copies of the protograph are randomly connected by edge permutations. Each non-zero entry of the corresponding base matrix $\mathbf{B}$ represents the number of connected edges to this node type. For further details we refer interested readers to [15], [16].

For the sake of spatial coupling, $\mathbf{B}$ can be divided into $W$ sub-matrices $\mathbf{B}_{i}$ of dimension $M^{\prime} \times N^{\prime}$ [15], i.e.,

$$
\mathbf{B}=\left[\begin{array}{c}
\mathbf{B}_{0} \\
\vdots \\
\mathbf{B}_{W-1}
\end{array}\right]_{W M^{\prime} \times N^{\prime}} .
$$

The approach from [15] is used to construct the SC-LDPC protograph matrix $\mathbf{B}_{\mathbf{L}, \mathbf{W}}$, where $L$ denotes the replication factor (i.e., the number of sub-blocks). For a terminated code and a coupling window $W=3$, we get

$$
\mathbf{B}_{L, W=3}=\left[\begin{array}{cccc}
\mathbf{B}_{0} & & & \\
\mathbf{B}_{1} & \mathbf{B}_{0} & & \\
\mathbf{B}_{2} & \mathbf{B}_{1} & \ddots & \\
& \mathbf{B}_{2} & \ddots & \mathbf{B}_{0} \\
& & \ddots & \mathbf{B}_{1} \\
& & & \mathbf{B}_{2}
\end{array}\right]_{(L+W-1) M^{\prime} \times L N^{\prime}}
$$

Finally, a lifting step with lifting factor $Z$ results in the parity-check matrix $\mathbf{H}_{L, W, Z}$.

\section{A. Density Evolution for the GMAC with iterative detec- tion/decoding}

The decoding threshold can be obtained via density evolution [16], [17]. We apply a Gaussian approximation (GA), i.e., we only track the mean value $\mu$ of messages passed along within the decoder and the iterative MUD with transfer function $f_{\mathrm{MUD}}\left(\mu_{\mathrm{A}, \mathrm{MUD}}\right)$ as in [10].

We denote the entry of $\mathbf{B}_{L, W}$ in the $j$-th row and the $i$-th column as $B_{j, i}$. Let $\mu_{i \leftarrow j}$ denote the mean value of messages passed from check node decoder (CND) $c_{j}$ with spatial position $j$ to a connected variable node decoder (VND) $v_{i}$ at spatial position $i$ and let $\mu_{i \rightarrow j}$ denote the mean value passed from VND $v_{i}$ to CND $c_{j}$. The update rules become ${ }^{1}$ [10], [17]

$$
\begin{aligned}
\mu_{i \leftarrow j}=\phi^{-1}(1- & {\left[1-\phi\left(\mu_{i \rightarrow j}\right)\right]^{B_{j, i}-1} } \\
\cdot \prod_{k=1 ; k \neq i}^{L N^{\prime}}\left[1-\phi\left(\mu_{i \rightarrow j}\right)\right]^{B_{j, k}} &
\end{aligned}
$$

with $\phi(\mu)$ as in [17]

$\phi(x)= \begin{cases}1-\frac{1}{\sqrt{4 \pi x}} \int_{-\infty}^{\infty} \tanh \left(\frac{u}{2}\right) \exp \left(-\frac{(u-x)^{2}}{4 x}\right) \mathrm{d} u, & x>0 \\ 1, & x=0\end{cases}$

Due to the serial concatenation of a REP of rate $R_{r}=\frac{1}{d_{r}}$, the mean of the messages passed from VND $v_{i}$ (including the REP code) to the MUD is therefore

$$
\mu_{D \leftarrow i}=\left(d_{r}-1\right) \mu_{D \rightarrow i}+\sum_{k=1}^{L M^{\prime}} B_{k, i} \cdot \mu_{i \leftarrow k} .
$$

Here $\mu_{D \rightarrow i}$ denotes the mean of the message from MUD to VND $v_{i}$ after the PIC processing at MUD nodes. These updated messages can be written as [9]

$$
\mu_{D \rightarrow i}=\frac{4}{N \sigma_{n}^{2}+(N-1) \cdot \phi\left(\mu_{D \leftarrow i}\right)} .
$$

\footnotetext{
${ }^{1}$ For readability, we only consider edges where $B_{j, i} \neq 0$, all unconnected edges $\left(B_{j, i}=0\right)$ virtually transmit $\mu=0$.
} 
TABLE I

DENSITY EVOLUTION-BASED DECODING THRESHOLDS OF DIFFERENT CODES FOR 8 USERS OVER THE GMAC AND $d_{r}$ SUCH THAT $R_{s u m}=1$ AND THE SHANNON LIMIT $\gamma_{\mathrm{Sh}}=0 \mathrm{~dB}$

\begin{tabular}{ccccc}
$d_{r}$ & $d_{v}$ & $d_{c}$ & uncoupled $\left(\gamma_{\mathrm{un}}^{*}\right)$ & coupled $^{2}\left(\gamma_{\mathrm{SC}}^{*}\right)$ \\
\hline \hline 4 & 3 & 6 & $2.54 \mathrm{~dB}$ & $1.55 \mathrm{~dB}$ \\
4 & 4 & 8 & $3.43 \mathrm{~dB}$ & $1.42 \mathrm{~dB}$ \\
4 & 5 & 10 & $4.11 \mathrm{~dB}$ & $1.33 \mathrm{~dB}$ \\
4 & 6 & 12 & $4.62 \mathrm{~dB}$ & $1.13 \mathrm{~dB}$ \\
\hline 2 & 3 & 4 & $3.96 \mathrm{~dB}$ & $0.74 \mathrm{~dB}$ \\
2 & 6 & 8 & $14.98 \mathrm{~dB}$ & $0.69 \mathrm{~dB}$ \\
2 & 9 & 12 & - & $0.69 \mathrm{~dB}$
\end{tabular}

The variable node update from VND $v_{i}$ to CND $c_{j}$ is

$\mu_{i \rightarrow j}=d_{r} \mu_{D \rightarrow i}+\left(B_{i, j}-1\right) \cdot \mu_{i \leftarrow j}+\sum_{k=1, k \neq j}^{L M^{\prime}} B_{k, i} \cdot \mu_{i \leftarrow k}$.

In this work, we use codes as proposed in [15]:

- $C_{1}$ : SC-LDPC $\left(d_{v}=3, d_{c}=6, L, W=3\right)$ code with $R_{c, L \rightarrow \infty}=0.5$ and $\mathbf{B}_{0}=\mathbf{B}_{1}=\mathbf{B}_{2}=\left[\begin{array}{ll}1 & 1\end{array}\right]$

- $C_{2}$ : SC-LDPC $\left(d_{v}=3, d_{c}=4, L, W=2\right)$ code with $R_{c, L \rightarrow \infty}=0.25$,

$$
\mathbf{B}_{0}=\left[\begin{array}{llll}
1 & 1 & 0 & 0 \\
0 & 1 & 1 & 0 \\
0 & 0 & 1 & 1
\end{array}\right] \quad \text { and } \quad \mathbf{B}_{1}=\left[\begin{array}{llll}
0 & 0 & 1 & 1 \\
1 & 0 & 0 & 1 \\
1 & 1 & 0 & 0
\end{array}\right] \text {. }
$$

Table I shows the decoding thresholds $\gamma_{\mathrm{un}}^{*}$ and $\gamma_{\mathrm{sc}}^{*}$ for the uncoupled and the SC-LDPC ensemble, respectively. As expected a higher node degree degrades the uncoupled thresholds, however, the SC-LDPC codes show an improved threshold which coincides well with the effect of threshold saturation [12].

\section{B. EXIT analysis}

Fig. 2 shows the extrinsic information transfer (EXIT) chart for the proposed MUD system with a SC-LDPC code at an SNR of $\gamma=2.3 \mathrm{~dB}$, i.e., below the uncoupled decoding threshold of $\gamma_{\text {un }}^{*}=2.54 \mathrm{~dB}$. As it can be seen, convergence is possible although no open decoding tunnel exists in the EXIT chart (cf. micro-convergence in [10]). As successful decoding is possible even if VND and CND curves intersect, no extensive degree profile matching of the code components is required anymore. This intuitively visualizes the universal behavior of SC-LDPC codes as their decoding thresholds are (approximately) universal for varying channel conditions [10], [11].

\section{WINDOWED MUD AND CHANNEL DECODER}

So far, we considered full BP decoding, meaning that all spatial positions are updated in parallel for each iteration. However, the authors in [13] showed that a windowed decoding scheme reduces decoding complexity without significant bit error rate (BER) performance loss. Throughout this work, we assume a constant number of iterations per window shift, yet, an adaptive implementation is possible [18].

\footnotetext{
${ }^{2}$ Remark: for a better comparison the rate loss due to termination effects is not considered here.
}

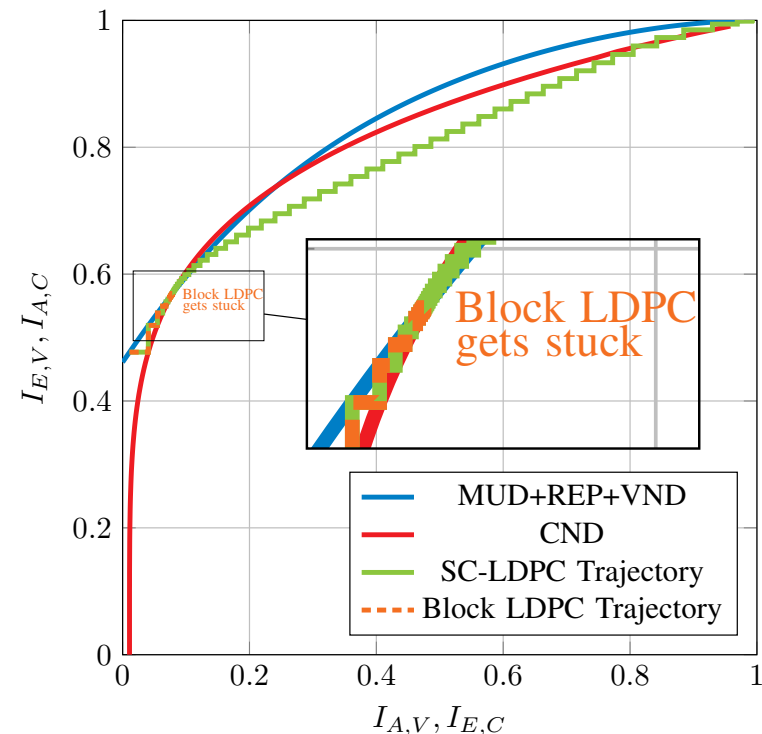

Fig. 2. EXIT chart of a $(3,6)$-SC-LDPC IDMA system at SNR $\gamma=2.3 \mathrm{~dB}$ and $d_{r}=4$ and 8 user, i.e., $R_{\text {sum }}=1$.

\section{A. Windowed receiver}

The windowed decoder makes use of the fact that the BER per spatial position converges in a wavelike manner, i.e., subsequent blocks can only be decoded if the previous blocks have been successfully decoded. Therefore, it is sufficient to only update nodes within a few spatial positions (active window), i.e., per decoding iteration only $W_{d} \geq W$ sub-blocks are active. We keep the decoding structure as in Fig. 1, but activate the same window for all users in parallel (i.e., all nodes at the same spatial positions). The windowed MUD estimates the SNR per sub-block (see Sec. IV-B for the intuition behind) and updates its outgoing messages accordingly. Finally, the window position is shifted by one spatial position after $I_{\max }$ decoding iterations until all sub-blocks are decoded. For further details on the initialization, see [18].

\section{B. Subblock interleaving}

The general idea of IDMA systems is to separate users by user-specific interleavers which are straightforward to implement for block-codes (besides complexity considerations). However, for SC-LDPC codes (and also due to the windowed receiver scheme) these interleavers require some further attention regarding the interleaving depth.

In (5), we assumed that the incoming messages from the MUD are all from spatial position $i$ for all users, i.e., they have the same underlying statistics and, thus, $\mu_{D \leftarrow i}$ is the same for all users. However, when assuming random interleaving over the whole $\mathbf{B}_{S C}$, the update in (5) changes to

$$
\tilde{\mu}_{D}=\tilde{\mu}_{D \leftarrow i}=\frac{1}{L N^{\prime}} \sum_{k=1}^{L N^{\prime}} \mu_{D \leftarrow k}
$$

and, thus, a full interleaver potentially destroys the locality of the SC-LDPC code and spatial positions with high interference noise (yet unconverged positions) hinder wavelike 


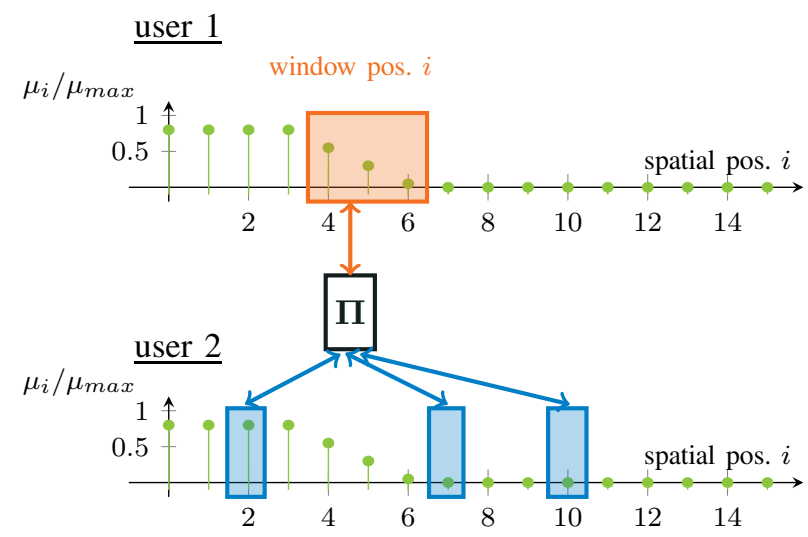

Fig. 3. Illustration of wavelike-decoding in a two-user IDMA system with a full interleaver accessing random spatial positions of user 2 that are not yet converged.

convergence. Further, the windowed MUD does not even update spatial positions outside the currently active window. Intuitively, this can be explained as illustrated in Fig. 3 for the two-user scenario. As the current decoding progress of spatial position $i$ shares messages with the other users, a random interleaver causes the access of random spatial positions (messages potentially not yet updated) of the other users. This means a user observes the high interference noise as if no (only little) a priori knowledge at the receiver exists. Thus, the overall performance can be approximated by a non-iterative scheme, where the initial estimate of the MUD provides a lower bound on its performance, i.e., $\tilde{f}_{\mathrm{MUD}}\left(\mu_{\mathrm{A}, \mathrm{MUD}}\right) \approx f_{\mathrm{MUD}}(0)$.

To keep the spatial structure of the code, we propose to use sub-block interleavers which only permute locally within a spatial position. This suffices for the required user separation, but still maintains the locality of the SC-LDPC code. However, the price to pay is a potentially smaller interleaver size (or larger sub-block size) and, thus, a slightly degraded BER performance of the IDMA system. Fig. 4 compares the BER performance for a system with the proposed sub-block interleaver (simulation parameters in Sec. V). It can be seen that the decoding performance under full interleaving together with windowed decoding degrades to the expected non-iterative performance, i.e., each user sees the SINR $\frac{1}{(N-1)+N \sigma_{n}^{2}}$ while the sub-block interleaving yields results close to $\gamma_{\mathrm{SC}}^{*}$ as provided in Table I. If full BP decoding is used with full interleaving, the performance can be dramatically enhanced with 1500 allowed iterations, but is still worse than the subblock interleaved version with windowed decoding.

\section{Simulation Results}

We consider an IDMA system with $N=8$ users and a targeted sum-rate of $R_{\text {sum }}=N \frac{R_{c}}{d_{r}}=1$. Both, AWGN and Rayleigh fading channel models are considered. For the AWGN channel case, an explicit phase scrambling is included into the symbol-mapper to improve the distance between multi-user superimposed codewords [14]. For Rayleigh fading channels, we consider that the channel states are uncorrelated

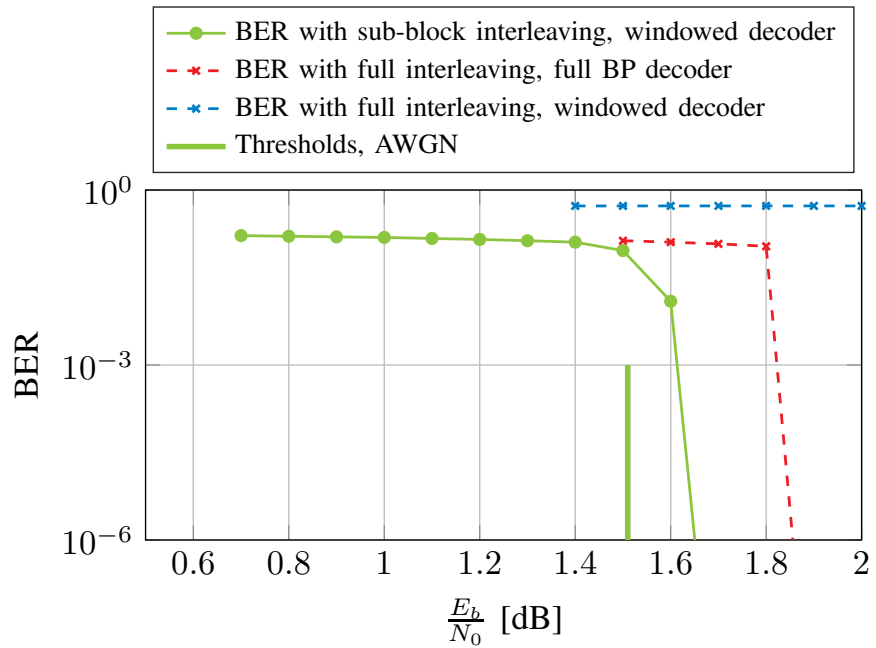

Fig. 4. BER performance of the SC-LDPC $\left(d_{v}=3, d_{c}=6\right)$ IDMA system with 8 users and $d_{r}=2$ for different interleaver implementations.

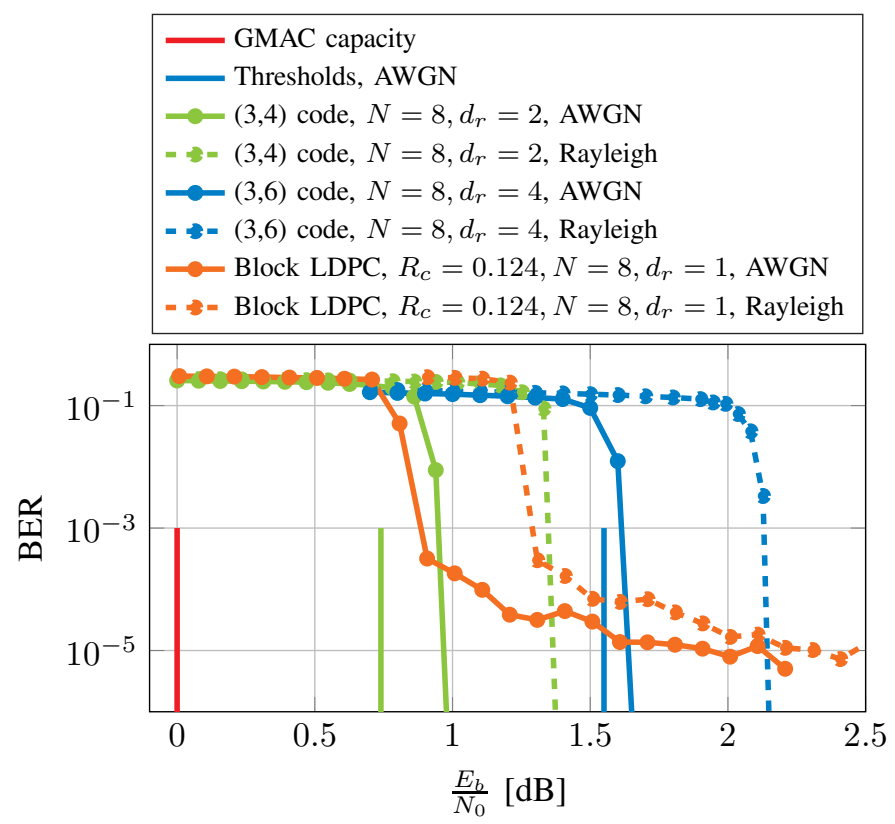

Fig. 5. BER performance of SC-LDPC-coded IDMA systems with window decoder in AWGN and Rayleigh fading channels; $N=8$ users with equalpower and equal-rate are considered.

among users and vary rapidly from symbol to symbol (ergodic fading, i.e., uncorrelated fast fading) and, therefore, a phase scrambler is not necessary in Rayleigh channels. Furthermore, the received signal power levels are assumed to be the same, i.e., $P_{i}=\frac{1}{N}, \forall i$ and BPSK is used as modulation format. For the unequal-power case, the repetition code can be used as "power equalizer" (see [9]).

Fig. 5 shows the BER results for such SC-LDPC-coded IDMA systems with the windowed detection and decoding scheme as described in Sec. IV. In the simulations, we use a decoding window length of $W_{d}=10$ sub-blocks, each consisting of 8000 symbols; $I_{\max }=40$ iterations are carried out per window shift. As provided in Sec. III-A, the code construction $C_{1}$ with $d_{v}=3, d_{c}=6, d_{r}=4, W=3$ and the construction $C_{2}$ with $d_{v}=3, d_{c}=4, d_{r}=2, W=2$ are 


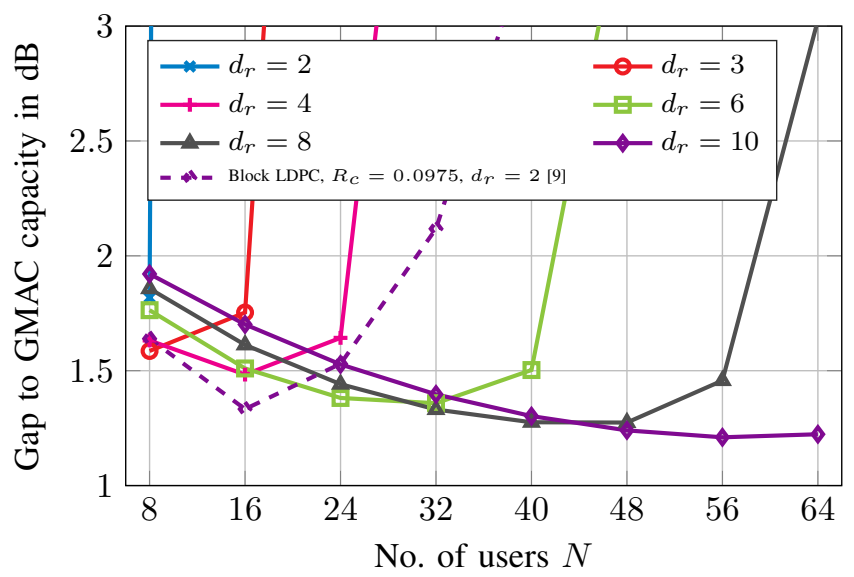

Fig. 6. DE-based thresholds of the SC-LDPC $\left(d_{v}=3, d_{c}=6, L, W=3\right)$ code $C_{1}$ with varying number of users $N$ and repetition factor $d_{r}$.

considered (for sum-rate one) and the total codeword length of the serially concatenated SC-LDPC and repetition code is fixed to $N_{\mathrm{CW}}=4 \cdot 10^{5}$. The simulated BERs for both cases at $10^{-6}$ in AWGN channel are about $0.2 \mathrm{~dB}$ larger than the decoding thresholds obtained from density evolution. The gap to the ultimate GMAC capacity is about $1 \mathrm{~dB}$ and $1.7 \mathrm{~dB}$ for the $(3,4)$ and $(3,6)$ codes, respectively. Compared to the AWGN channel case, the performance loss due to Rayleigh fading is only $0.4 \mathrm{~dB}$ for both codes, due to the so-called multiuser diversity in fading channels [3]. The performance of block LDPC code (code-word length is $N_{\mathrm{CW}}=4 \cdot 10^{5}$, same as SCLDPC codes) with EXIT chart based matching degree profiles is included for comparison. Besides the cumbersome matching procedure, the required irregularity in the parity-check matrix unfortunately degrades the error-floor performance of the code.

As the number of users in a MAC system can vary, we propose to use the additional REP to cope with the varying number of users [9]. Fig. 6 shows the GA DE-based gapto-capacity results for various repetition codes and number of users, while for all those curves the used SC-LDPC $\left(d_{v}=3, d_{c}=6, L, W=3\right)$ code $C_{1}$ is fixed. It can be observed that increasing the repetition factor $d_{r}$ leads to a quite universal support over a wide range of number of users $N$. For instance with $d_{r}=10$, the gap-to capacity can be kept below $2 \mathrm{~dB}$ for the number of users in the range $N \in[8,64]$. For comparison, the results for a block LDPC code with optimized degree profile are also included, where the block LDPC code is of the rate $R_{c}=0.0975 \approx 0.1$. With a further repetition code of $d_{r}=2$, the total code rate is the same as the $(3,6)$ SC-LDPC code with $d_{r}=10$. It is obvious that (if fixed) the SC-LDPC code can support a wider range of users, although the gap-to-capacity can be larger for some number of users.

\section{CONCLUSIONS AND OUTLOOK}

We have analyzed SC-LDPC codes as coding scheme for an IDMA multi-user system with a sliding windowed-based iterative detection and decoding receiver. We have examined the decoding thresholds through density evolution and shown that thresholds saturation occurs. The SC-LDPC codes benefit from the anticipated universal behavior of SC-LDPC codes with respect to the channel front-end and, thus, do not require explicitly matched degree profiles for a specific number of users or changing channel characteristic. It also relaxes potential error-floor issues in finite-length code design, as regular node degrees instead of highly irregular degree profiles are sufficient. Further, a windowed receiver implementation, consisting of both windowed detector and windowed decoder, keeps the overall decoding complexity within a feasible range but requires sub-block interleaving. As a result, the proposed system operates below $1 \mathrm{~dB}$ away from the GMAC capacity at a BER of $10^{-6}$ for finite length code constructions.

\section{REFERENCES}

[1] C. Berrou, A. Glavieux, and P. Thitimajshima, "Near Shannon limit error-correcting coding and decoding: Turbo-codes," Proc. IEEE Int. Conf. on Commun. (ICC), pp. 1064-1070, May 1993.

[2] S.-Y. Chung, G. D. Forney, T. J. Richardson, and R. Urbanke, "On the design of low-density parity-check codes within $0.0045 \mathrm{~dB}$ of the Shannon limit," IEEE Comm. Letters, vol. 5, no. 2, pp. 58-60, 2001.

[3] D. Tse and P. Viswanath, Fundamentals of Wireless Communications. Cambridge University Press, 2005.

[4] L. Dai, B. Wang, Z. Ding, Z. Wang, S. Chen, and L. Hanzo, "A survey of non-orthogonal multiple access for 5G," IEEE Commun. Surveys Tuts., vol. 20, no. 3, pp. 2294-2323, 2018.

[5] L. Dai, B. Wang, Y. Yuan, S. Han, C. I, and Z. Wang, "Non-orthogonal multiple access for $5 \mathrm{G}$ : solutions, challenges, opportunities, and future research trends," IEEE Commun. Mag., vol. 53, no. 9, pp. 74-81, Sep. 2015.

[6] L. Ping, L. Liu, K. Y. Wu, and W. K. Leung, "Approaching the capacity of multiple access channels using interleaved low-rate codes," IEEE Comm. Letters, vol. 8, no. 1, pp. 4-6, Jan 2004.

[7] L. Ping, L. Liu, K. Wu, and W. K. Leung, "Interleave division multipleaccess," IEEE Trans. Wireless Commun., vol. 5, no. 4, pp. 938-947, April 2006.

[8] S. ten Brink, G. Kramer, and A. Ashikhmin, "Design of low-density parity-check codes for modulation and detection," IEEE Trans. Commun., vol. 52, no. 4, pp. 670-678, April 2004.

[9] X. Wang, S. Cammerer, and S. t. Brink, "Near Gaussian multiple access channel capacity detection and decoding," arXiv preprint arXiv:1811.10938, 2018.

[10] L. Schmalen and S. ten Brink, "Combining spatially coupled LDPC codes with modulation and detection," in ITG Conference on Systems, Communication and Coding (SCC). VDE, 2013, pp. 1-6.

[11] S. Cammerer, L. Schmalen, V. Aref, and S. ten Brink, "Wave-like decoding of tail-biting spatially coupled LDPC codes through iterative demapping," in Proc. Internat. Symp. Turbo Codes, 2016, pp. 121-125.

[12] S. Kudekar, T. Richardson, and R. Urbanke, "Spatially coupled ensembles universally achieve capacity under belief propagation," IEEE Trans. Inform. Theory, vol. 59, no. 12, pp. 7761-7813, 2013.

[13] A. R. Iyengar, P. H. Siegel, R. Urbanke, and J. K. Wolf, "Windowed decoding of spatially coupled codes," IEEE Trans. Inform. Theory, vol. 59, no. 4, pp. 2277-2292, 2013.

[14] G. Song and J. Cheng, "Distance enumerator analysis for interleavedivision multi-user codes," IEEE Trans. Inform. Theory, vol. 62, no. 7, pp. 4039-4053, July 2016.

[15] D. Mitchell, M. Lentmaier, and D. J. Costello Jr., "Spatially coupled LDPC codes constructed from protographs," IEEE Trans. Inform. Theory, vol. 61, no. 9, pp. 4866-4889, Sept 2015.

[16] T. Richardson and R. Urbanke, Modern Coding Theory. Cambridge University Press, 2008.

[17] S.-Y. Chung, T. J. Richardson, and R. Urbanke, "Analysis of sumproduct decoding of low-density parity-check codes using a Gaussian approximation," IEEE Trans. Inform. Theory, vol. 47, Feb 2001.

[18] K. Klaiber, S. Cammerer, L. Schmalen, and S. ten Brink, "Avoiding burst-like error patterns in windowed decoding of spatially coupled LDPC codes," in Proc. Internat. Symp. Turbo Codes, 2018. 